\title{
L'INFLUENCE DES RÉSIDENCES SECONDAIRES SUR L'ENVIRONNEMENT NATUREL ET LE DÉVELOPPEMENT LOCAL
}

Un grand développement de résidences secondaires aussi bien dans les régions les plus intéressantes du point de vue de la récréation que dans la zone péri-urbaine de grandes agglomérations entraîne, d'un côté, les transformations défavorables de l'environnement naturel mais de l'autre stimule les structures socio-économiques locales devenant ainsi un facteur important du développement local

L'expansion incontrôlée de résidences secondaires en Pologne est à l'origine du fait que la qualité de l'environnement naturel dans certaines régions a sensiblement baissé. N'oublions pas, pourtant, qu'il y a des régions où la crise a tout particulièrement touché la campagne. Ainsi, les régions du Nord et de l'Ouest de la Pologne où prédominaient les fermes d'État, de même que celles de l'Est du pays où le niveau d'agriculture est plutôt bas présentent le taux de chômage atteignant $20-30 \%$; ces régions se caractérisent par la baisse la plus importante des revenus individuels, et par la crise qui a touché les budgets des autorités locales. Afin de pallier à ce phénomène, le gouvernement a décidé, en 1992, de promouvoir le développement du tourisme dans les territoires ruraux, ce qui devrait contribuer à une augmentation des revenus aussi bien individuels que d'autorités locales, à la création de nouveaux postes de travail, et à la stimulation du développement du commerce et des services. Il sied pourtant de souligner qu'il ne s'agit pas là, du développement du tourisme en masse, à caractère commercial car, étant donné l'influence nocive de celui-ci et en vue de l'élimination de ses effets nuisibles, les spécialistes essaient de promouvoir le développement de l'éco-tourisme et de l'agro-tourisme. Dans ces circonstances, les résidences secondaires pourraient devenir un facteur supplémentaire stimulant le développement local dans les régions en crise, mais l'implantation de ces résidences devrait être soumise à un contrôle rigoureux effectué par les autorités locales aussi bien que par des spécialistes d'aménagement de l'espace. 
LES RÉSIDENCES SECONDAIRES ET LES STRUCTURES ÉCOLOGIQUES LOCALES

Il est difficile de déterminer l'influence d'habitations de récréation sur l'environnement naturel, étant donné que celles-ci coexistent avec d'autres formes d'utilisation du sol dont l'agriculture, les habitations à caractère permenent, ou les installations touristiques au sens strict du mot. L'estimation de dangers menaçant l'environnement naturel, provoqués par l'implantation de résidences secondaires, est d'autant plus difficile que les données sur l'équipement technique de celles-ci, l'émission de polluants, l'utilisation de l'eau, la dégradation des sols et de la végétation, de même que sur le nombre réel de ces résidences et la fréquence de leur utilisation font, le plus souvent, défaut.

Pourtant, même les observations des plus superficielles démontrent qu'une concentration trop dense de résidences secondaires entraîne l'abaissement de la nappe aquifere, des transformations de la couverture végétale, elle stimule les processus d'érosion des sols, enfin augmente le degré de la pollution chimique aussi bien des eaux de surface et des eaux souterraines que de l'air atmosphérique. Les dangers menaçant les structures écologiques, provoqués par les résidences secondaires, sont à redouter spécialement dans les localités où le nombre de ces résidences est particulièrement élevé, où l'infrastructure demeure inadaptée aux réalités nouvelles, et où la résistance de l'environnement naturel est plutôt basse. Ainsi, les nouveaux plans d'aménagement de l'espace qui seraient conçus en vue de la récréation devraient prendre ladite résistance en considération.

Afin de minimiser les effets négatifs que l'expansion de l'habitat de loisir apporte à l'environnement naturel, on a adopté le principe que la superficie minimum d'une parcelle de récréation devrait être de $700-800 \mathrm{~m}^{2}$. Comme une densité excessive de la construction de récréation exerce non seulement une influence nuisible sur les structures écologiques locales mais en même temps diminue la qualité du repos dans la résidence secondaire, il serait particulièrement important de propager les informations sur les interdépendances du mouvement de récréation et de la qualité du repos.

Néanmoins, les recherches effectuées en 1991 dans les communes de la banlieue varsovienne, et notamment à Magnuszew, Pomiechówek et Wiązowna, ont démontré qu'en général les propriétaires des résidences secondaires ne considèrent pas une densité excessive d'habitat de loisir comme un facteur ayant une influence nocive sur l'environnement naturel.

Parmi toutes les personnes interrogées dans les trois communes citées plus haut, il n'y avait que $10,7 \%$ estimant que les résidences secondaires peuvent exercer une influence nocive sur l'environnement naturel $(1,4 \%$ en sont convaincus) contre $54,4 \%$ qui considéraient cette influence comme neutre. Les propriétaires de résidences secondaires estimant que l'influence des fonctions de récréation sur les structures écologiques locales est 
négative étaient ceux de la commune de Magnuszew. Ainsi, une partie de ces propriétaires, installés dans le village de Mniszewo appartenant à ladite commune, sont d'avis que l'implantation des résidences secondaires entraîne la pollution de l'environnement et l'abaissement aussi bien de la nappe aquifere dans le bras mort de la Pilica que des eaux souterraines.

Tableau 1

Estimation de l'influence des résidences secondaires sur l'environnement naturel local

\begin{tabular}{|l|c|c|c|c|}
\hline & \multicolumn{4}{|c|}{ Communes } \\
\cline { 2 - 5 } & $\begin{array}{c}\text { Magnuszew } \\
(\mathbf{N}=177)\end{array}$ & $\begin{array}{c}\text { Pomiechówek } \\
(\mathbf{N}=205)\end{array}$ & $\begin{array}{c}\text { Wiazzowna } \\
(\mathbf{N = 1 9 9 )}\end{array}$ & $\begin{array}{c}\text { Total } \\
(\mathbf{N}=581)\end{array}$ \\
\hline Défavorable & 1,7 & 1,5 & 1,0 & 1,4 \\
Plutôt défavorable & 11,0 & 8,0 & 9,6 & 9,3 \\
Plutôt neutre & 39,9 & 36,0 & 29,2 & 34,9 \\
Neutre & 47,4 & 54,5 & 60,3 & 54,4 \\
\hline
\end{tabular}

Le plus souvent, les propriétaires en question découvraient tout d'abord les transformations défavorables dues à la pollution des eaux de surface, ensuite la dégradation de la couverture végétale et l'atteinte à la beauté du paysage. Il n'y a que dans la commune de Magnuszew que l'on citait la pollution de l'air atmosphérique en deuxième place après celle des eaux de surface (vu la proximité de la centrale électrique de Świerże Górne). La pollution de l'air préoccupait tout particulièrement les propriétaires installés dans le village de Kłoda qui, à leur tour, attribuaient à la centrale électrique de Kozienice (située à $5 \mathrm{~km}$ du groupement de résidences secondaires) la responsabilité de l'état de l'air.

Tableau 2

Transformations de l'environnement naturel observées par les propriétaires des résidences secondaires

\begin{tabular}{|l|c|c|c|c|}
\hline \multirow{2}{*}{ Transformations } & \multicolumn{4}{|c|}{ Communes } \\
\cline { 2 - 5 } & $\begin{array}{c}\text { Magnuszew } \\
(\mathrm{N}=177)\end{array}$ & $\begin{array}{c}\text { Pomiechówek } \\
(\mathrm{N}=205)\end{array}$ & $\begin{array}{c}\text { Wiazowna } \\
(\mathrm{N}=199)\end{array}$ & $\begin{array}{c}\text { Total } \\
(\mathrm{N}=581)\end{array}$ \\
\hline Eaux de surface & 36,7 & 33,2 & 22,1 & 30,5 \\
Eaux souterraines & 5,7 & - & 2,0 & 2,4 \\
Air atmosphérique & 13,0 & 1,5 & 2,0 & 5,2 \\
Couverture végétale & 9,0 & 8,3 & 20,1 & 12,6 \\
Faune locale & 2,0 & - & 1,5 & 0,9 \\
Paysage & 3,4 & 11,2 & 10,1 & $\mathbf{8 , 4}$ \\
\hline
\end{tabular}


Il serait injuste d'affirmer que l'unique cause de la dégradation de l'environnement naturel est l'absence de l'infrastructure nécessaire ou encore le comportement des citadins venus en villégiature. Citons plutôt un nombre trop grand de résidences secondaires (au même titre que d'autres éléments d'aménagement touristique), une petite superficie de parcelles et, ce qui s'ensuit, un nombre important d'usagers.

Selon l'enquête effectuée, il n'y a que $17 \%$ de personnes interrogées qui reconnaissent que le nombre de parcelles de récréation est trop grand dans un terrain donné. Mais d'autre part, les propriétaires de résidences en question, situées dans des terrains à la grande densité d'habitat, voient rarement la relation de cause à effet entre les dimensions d'un groupement d'habitations de récréation, la densité de l'habitat de loisir et l'état de l'environnement naturel. Espérons, pourtant, qu'au fur et à mesure du développement de la conscience écologique de la société, les propriétaires de résidences secondaires, eux aussi, adopteraient des attitudes pro-écologiques. Les populations et les autorités locales devraient adopter les mêmes attitudes et renoncer à l'idée de lotissement des terrains ruraux en parcelles, dans l'espoir d'en tirer des bénéfices plus importants que ceux de l'utilisation agricole du sol. Vient donc le tour d'urbanistes et d'architectes qui, en élaborant les projets d'aménagement soit d'espaces ruraux (commandés par les autorités locales), soit de parcelles individuelles (commandés par des particuliers) adopteraient les principes de l'éco-urbanisme. Il en résulte, d'expériences en la matière des pays développés, tels que les pays scandinaves, qu'avec le temps, les propriétaires de résidences secondaires commencent à coopérer avec les communautés locales en vue de la protection de l'environnement dans les territoires aux valeurs récréatives et touristiques particulières, en obligeant ainsi les autorités locales à entreprendre des démarches nécessaires dans ce sens (Christiansen et allii, 1979)

\section{LES RÉSIDENCES SECONDAIRES ET L'ÉCONOMIE LOCALE}

On admet, en général, que l'expansion de l'habitat de loisir dans les terrains ruraux accélère les transformations de la structure agraire, impose l'intensification de l'agriculture, favorise le développement du commerce et des services, ouvre les possibilités de la création de nouveaux emplois augmente les revenus des populations et d'autorités locales, ensuite exerce une influence favorable sur l'état de l'habitat, entre autres en freinant la dépopulation qui résulte de l'émigration (parfois même en initiant l'immigration) et des processus de vieillissement des populations, enfin contribue à une renovation des habitations appartenant aux villageois (Coppock 1977)

Une influence stimulante de résidences secondaires sur l'économie locale 
est visible surtout dans les terrains qui, avant, connaissaient une dépression économique, qui se dépeuplaient, terrains à l'agriculture en déchéance, éloignés, le plus souvent, des grandes agglomérations urbaines et industrielles. Ce rôle stimulant se réduit principalement à une augmentation de revenus grâce aux taxes et impôts payés par les propriétaires des résidences secondaires. De l'avis de D.Clary (1977), chercheur français qui étudiait la condition de communes normandes, les revenus obtenus grâce à l'installation des résidences secondaires sont plus élevés que les revenus rapportés par les propriétaires d'autres habitations. On observe le même phénomène en Pologne où, depuis les élections locales de 1990 , les conseils communaux établissent les impôts sur les parcelles et maisons de récréation au niveau plus élevé que dans le cas de biens immeubles appartenant aux habitants mêmes de la commune. Ainsi, dans les régions où le nombre de résidences secondaires est important, les revenus obtenus des propriétaires de celles-ci commencent à dépasser les revenus d'autres impôts et deviennent par là-même le revenu principal de la commune (Kowalczyk 1991). Citons ici les communes de Jadów (voïvodie de Siedlce) et de Mielnik (voïvodie de Białystok) où, en 1992, l'impôt établi sur $1 \mathrm{~m}^{2}$ de superficie dans une résidence secondaire dépassait de vingt fois l'impôt sur $1 \mathrm{~m}^{2}$ de superficie dans une habitation permanente. Mais les initiatives de ce genre - en dépit de bénéfices immédiats - pourraient décourager dans l'avenir d'éventuels acheteurs de parcelles dans une commune donnée et, par là-même, freiner le développement de fonctions récréatives. Dans la situation de crise de l'agriculture, et étant donné la nécessité de transformations, l'adoption d'une politique de ce genre augmenterait encore la dégradation de la campagne polonaise et rendrait difficile, sinon impossible, la transition du modèle à la fonction unique en modèle plurifonctionnel.

Un autre aspect - fréquemment exposé - du développement du phénomène des résidences secondaires dans les terrains de réception est une augmentation de revenus des populations locales. Cette augmentation de revenus s'effectue grâce à la vente de biens immeubles aux futurs propriétaires de résidences secondaires, ensuite grâce à une animation sur le marché du travail et à l'accroissement de la demande de produits locaux.

Il y a déjà plus de dix ans qu'on s'était rendu compte du rôle stimulant que les résidences secondaires jouent dans les économies locales. Les études empiriques démontrent que l'influence positive que ces résidences exercent sur les transformations socio-économiques est appréciée à sa juste valeur. Citons en exemple les communes, choisies au hasard, de la banlieue varsovienne. Or, si en 1979, dans la commune de Wilga, $34,5 \%$ de personnes interrogées estimaient que les résidences secondaires constituent un phénomène avantageux à la commune (Kowalczyk 1988), en 1992, 83,5\% de personnes interrogées dans la commune de Radzymin étaient du même avis. Ceci témoigne du changement de l'attitude à l'endroit de résidences 
secondaires et du rôle stimulant qu'elles jouent dans les économies locales. On a apprécié surtout l'influence desdites résidences sur l'accroissement des revenus individuels d'agriculteurs $(95,6 \%)$ et l'amélioration de l'état du budget communal $(81,9 \%)$. Moins nombreux étaient les habitants qui constataient l'influence positive des fonctions récréatives sur le développement culturel des villages $(49,2 \%)$, l'amélioration de l'état de voies locales, et le fonctionnement des transports publics (48\%). L'influence positive de résidences secondaires a été reconnue, le plus souvent, par les habitants ayant déjà vendu des terres aux propriétaires de ces résidences, et par ceux qui estiment les rapports entre les usagers de parcelles et les populations locales comme fréquents.

L'installation de résidences en question joue un rôle important sur le marché local de construction. En dépit d'avantages incontestables qu'elles présentent aux yeux d'entrepreneurs locaux, ces résidences sont à l'origine des conflits point négligeables entre leurs propriétaires d'un côté et les populations locales, de l'autre. Le pouvoir d'achat de villageois est, d'habitude, plus faible que celui de propriétaires de résidences, ainsi les habitants sont incapables de faire face à la concurrence créée par les nouveaux venus dont la présence engendre, il est vrai, un "boom de construction" mais de l'autre côté, fait monter les prix (vu l'accroissement de la demande dans les conditions où l'offre des services de construction est, le plus souvent, limitée).

N'oublions pas, enfin, que l'installation de résidences secondaires grâce à l'argent qu'elles rapportent aux budgets locaux - peut contribuer à un développement de l'infrastructure technique (voies, réseaux de distribution de l'eau, téléphones) en améliorant ainsi le niveau de vie de la campagne ce qui est un phénomène hautement positif et un facteur important du développement local.

En récapitulant, nous pouvons constater que si l'influence de résidences secondaires sur les structures socio-économiques locales est, le plus souvent, positive, par contre l'influence qu'elles exercent sur l'environnement naturel est plutôt défavorable. Ce n'est donc qu'en limitant leur concentration dans des terrains où elle devient excessive, en installant des dispositifs techniques permettant de minimiser la dégradation de l'environnement et, enfin, en inculquant aux usagers les attitudes proécologiques que l'on pourrait en faire un facteur incontestablement positif du développement local.

\section{BIBLIOGRAPHIE}

Christiansen P.B., Lundgaard B., Olesen O., 1979, 'Work, Leisure and Planning ", dans: Arbejdsrapport, 8, Geografisk Institut, Aarhus Universitet, pp. 1-24

Clary D., 1973, "Les résidences secondaires: approche financière du problème. 
L'exemple de la côte normande", Bulletin de la Société languedocienne de géographie, 7, 3/4, pp.321-332.

C op pock J.T., 1977, "Second Homes in Perspective", dans: Coppock J.T.(éd.) Second Homes: Curse or Blessing, 7, Pergamon Press, pp.1-15.

Kowalczyk A., 1988, "Second Homes as a Research Subject in Social Geography", dans: Otok, S. (éd.), Research Approach to Social Geography, Faculty of Geography and Regional Studies, University of Warsaw, pp.133-142.

Kowalczyk A., 1991, "Trzy postulaty badawcze dla polskiej geografii turyzmu lat dziewięćdziesiątych" (Trois propositions de recherches adressées à la géographie polonaise du tourisme des années quatre-vingt-dix), Turyzm, 2, pp.56-61m. 
